\title{
AFFINE INVARIANT TEXTURE SIGNATURES ${ }^{1}$
}

\author{
Jianguo Zhang, Tieniu Tan \\ National Laboratory of Pattern Recognition (NLPR), \\ Institute of Automation, Chinese Academy of Sciences, Beijing, 100080, P.R.China \\ \{jgzhang, tnt\}@nlpr.ia.ac.cn
}

\begin{abstract}
In this paper, we develop a new approach for texture classification independent of affine transforms. Based on spectral representation of texture images under affine transform, anisotropic scale invariant signatures of orientation spectrum distribution are extracted. Peaks distribution vector (PDV) obtained on the distribution of these signatures captures texture properties invariant to affine distortion. The PDV is used to measure the similarity between textures. Experimental results show the efficiency of the PDV for affine invariant texture classification.
\end{abstract}

\section{INTRODUCTION}

Texture analysis is a key issue in image processing, computer vision and their applications, such as object recognition, remote sensing, content-based image retrieval and so on. It has been an active research topic for more than three decades. Numerous methods have been proposed in the open literature. The majority of existing methods make the explicit or implicit assumptions that texture images are acquired from the same viewpoint (e.g. the same scale and orientation). However in many practical applications such as content-based viewpoint image retrieval, images obtained are often subject to geometric distortions including translation, rotation, scaling and skew. So texture features should ideally be invariant to viewpoint.

More and more attention has been paid on invariant texture analysis and a great deal of work has been performed on this important subject. But most of the existing invariant texture features are only invariant to translation, rotation or scaling [1][6]. Few work deals with invariant texture analysis under affine or perspective transform [7][9].

In this paper we focus on affine invariant texture analysis. Spectral representation of texture images under affine transform is first derived and then normalized to mathematically satisfy affine model. Anisotropic scale invariant signatures of orientation spectrum distribution are extracted. Peaks distribution vector (PDV) obtained on the distribution of these signatures captures texture properties invariant to affine distortion.

\section{SPECTRAL REPRESENTATION OF TEXTURE UNDER AFFINE TRANSFORM}

It is well known that affine transform can be decomposed into scale, skew, rotation and translation. It can be viewed as the approximation of the perspective projection if the depth of an object is relatively large with respect to its dimension [2].

Let $f(x, y)$ be the original image and $f_{a}\left(x_{a}, y_{a}\right)$ the affine transformed version. Then the relationship of these two images is as follows:

$$
\begin{gathered}
f_{a}\left(x_{a}, y_{a}\right)=f(x, y) ; \\
{\left[\begin{array}{l}
x_{a} \\
y_{a}
\end{array}\right]=\left[\begin{array}{ll}
c_{11} & c_{12} \\
c_{21} & c_{22}
\end{array}\right]\left[\begin{array}{l}
x \\
y
\end{array}\right]+\left[\begin{array}{l}
d_{1} \\
d_{2}
\end{array}\right]}
\end{gathered}
$$

Let matrix $A$ denote $\left[\begin{array}{ll}c_{11} & c_{12} \\ c_{21} & c_{22}\end{array}\right]$ and $D$ $\left[\begin{array}{l}d_{1} \\ d_{2}\end{array}\right] . D$ is translation factor and $A$ is rotation and scaling factor. It is easy to see that when the original texture undergoes affine distortion, the frequency spectrum is also transformed by a similar set of transformations.

Let $F(u, v)$ be the Fourier transform of the original texture $f(x, y), F_{a}\left(u_{a}, v_{a}\right)$ the Fourier transform of $f_{a}\left(x_{a}, y_{a}\right)$. Thus we can obtain the following equations (here we assume that

\footnotetext{
1 This work is funded by research grants from the NSFC (Grant No. 69825105 and 69790080) and the Chinese Academy of Sciences.
} 


$$
\begin{aligned}
& \left.\operatorname{det}\left(A^{-1}\right) \neq 0\right)[2]: \\
& \left|F_{a}\left(u_{a}, v_{a}\right)\right|=\operatorname{det}\left(A^{-1}\right)|F(u, v)|,\left(\begin{array}{l}
u_{a} \\
v_{a}
\end{array}\right)=A_{T}^{-1}\left(\begin{array}{l}
u \\
v
\end{array}\right)
\end{aligned}
$$

where $A_{T}^{-1}$ is the inverse of the transpose of matrix A.

From Equation (2) and (1), we can see that the two expressions are very similar except that the spectrum in Equation (2) is scaled and the translation factor is removed. So that if Equation (2) is normalized by the sum of $|F(u, v)|$, the following equation can be derived:

$$
\frac{\left|F_{a}\left(u_{a}, v_{a}\right)\right|}{\sum\left|F_{a}\left(u_{a}, v_{a}\right)\right|}=\frac{|F(u, v)|}{\sum|F(u, v)|}
$$

Let $F_{a}^{\prime}\left(u_{a}, v_{a}\right)$ denote the left part of Equation (3) and $F^{\prime}(u, v)$ the right part. By rewriting Equation (3), we get

$$
\left|F^{\prime}{ }_{a}\left(u_{a}, v_{a}\right)\right|=\left|F^{\prime}(u, v)\right|,\left(\begin{array}{l}
u_{a} \\
v_{a}
\end{array}\right)=A_{T}^{-1}\left(\begin{array}{l}
u \\
v
\end{array}\right)
$$

Thus the normalized spectrum also satisfies the affine model. The relationship between textures and their spectral representations under affine transform is illustrated in Figure 1.

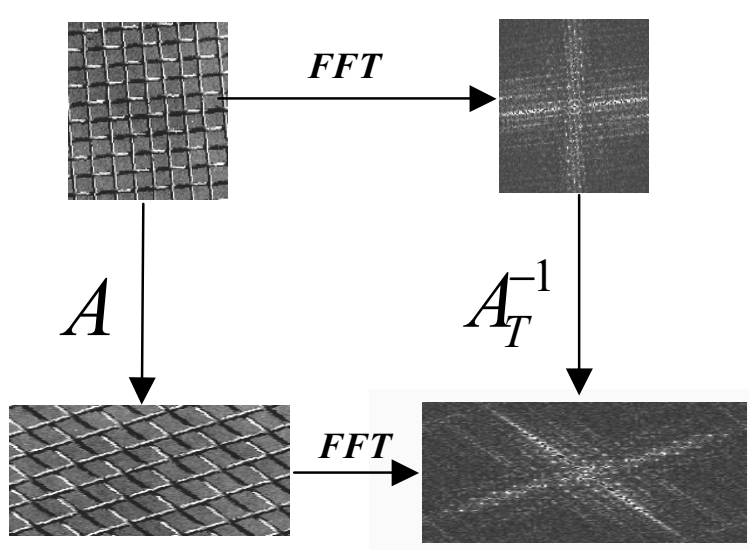

Figure 1 Spectral representation of textures under affine transform

\section{INVARIANT ORIENTATION SPECTRUM SIGNATURES}

In Section 2, we have shown that affine transform in the spatial domain results in similar affine transform in the spectrum. We have also derived a spectral representation
(Equation (4)) that mathematically satisfies affine model. This section discusses whether and how we can use the new representation for affine invariant texture analysis.

In order to simplify notations, here we simply use $F_{a}\left(u_{a}, v_{a}\right) \quad(F(u, v))$ to represent $\left|F_{a}^{\prime}\left(u_{a}, v_{a}\right)\right|$ $\left(\left|F^{\prime}(u, v)\right|\right)$, that is

$$
F_{a}\left(u_{a}, v_{a}\right)=F(u, v) ;\left(\begin{array}{l}
u_{a} \\
v_{a}
\end{array}\right)=\hat{A}\left(\begin{array}{l}
u \\
v
\end{array}\right)
$$

where $\hat{A}$ denotes the matrix $A_{T}^{-1}\left[\begin{array}{ll}\hat{c}_{11} & \hat{c}_{12} \\ \hat{c}_{21} & \hat{c}_{22}\end{array}\right]$.

Then we make some substitutions

$$
\begin{gathered}
\rho_{a}=\sqrt{u_{a}^{2}+v_{a}^{2}}, \theta_{a}=\operatorname{arctg}\left(u_{a} / v_{a}\right) \\
\rho=\sqrt{u^{2}+v^{2}}, \theta=\operatorname{arctg}(u / v)
\end{gathered}
$$

Equation (5) can be expressed in its polar version

$$
F_{a}\left(\rho_{a}, \theta_{a}\right)=F(\rho, \theta)
$$

where $F_{a}\left(\rho_{a}, \theta_{a}\right)$ and $F(\rho, \theta)$ are called the line-spread functions along the lines at angle $\theta_{a}$ and $\theta$. Let us investigate the changes between $\rho$ and $\rho_{a}$ by using their ratio as follows:

$$
\begin{gathered}
s=\frac{\rho_{a}}{\rho}=\frac{\sqrt{u_{a}^{2}+v_{a}^{2}}}{\sqrt{u^{2}+v^{2}}} \\
=\frac{\sqrt{\left(\hat{c}^{2}{ }_{11}+\hat{c}^{2}{ }_{21}\right) \operatorname{tg}^{2} \theta+\left(\hat{c}^{2}{ }_{12}+\hat{c}^{2}{ }_{22}\right)}}{\sqrt{\operatorname{tg}^{2} \theta+1}}
\end{gathered}
$$

The above equation indicates that $S$ is only dependent on $\theta$ for a given affine transform. Although $s$ varies with $\theta, s$ is a fixed value for a given orientation $\theta$. By an application of $\log$ operator on $\rho$ and $\rho_{a}$ along the orientation $\theta$ and $\theta_{a}$, we have

$$
F_{a}\left(w_{1}, \theta_{a}\right)=F\left(w_{2}, \theta\right)
$$

where

$$
\begin{gathered}
w_{1}=\log \rho_{a}=\log s+\log \rho \\
w_{2}=\log \rho
\end{gathered}
$$

It is important to note that in Equation (8) scaling in the line-spread spectrum function has been transformed into a shift by the log operation. Since the frequency distribution (here we consider spectrum magnitude as the probability of the corresponding frequency) can give a description of texture periodicity, we calculate the central moment of the spread line function as follows: 


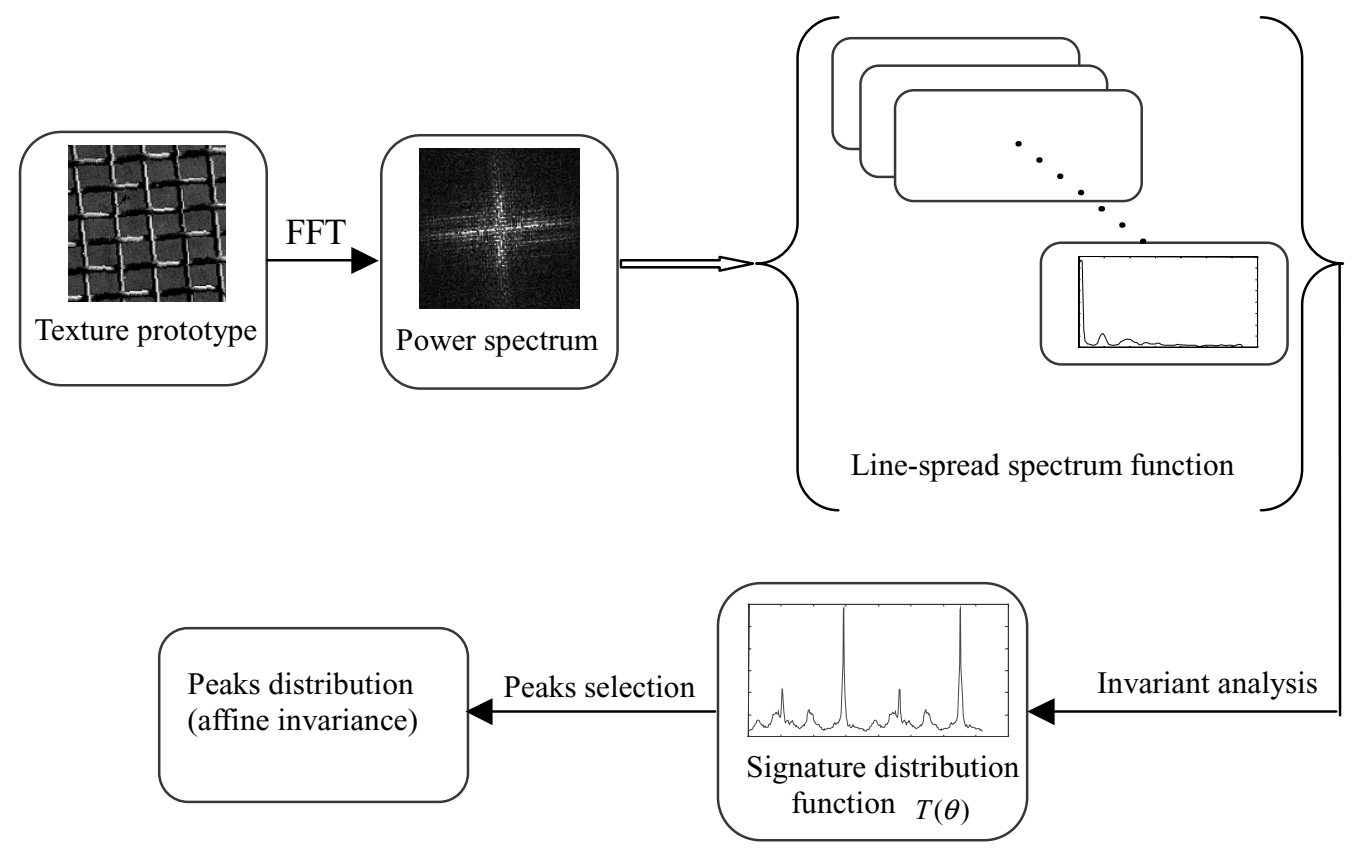

Figure 2 Global scheme of PDV extraction
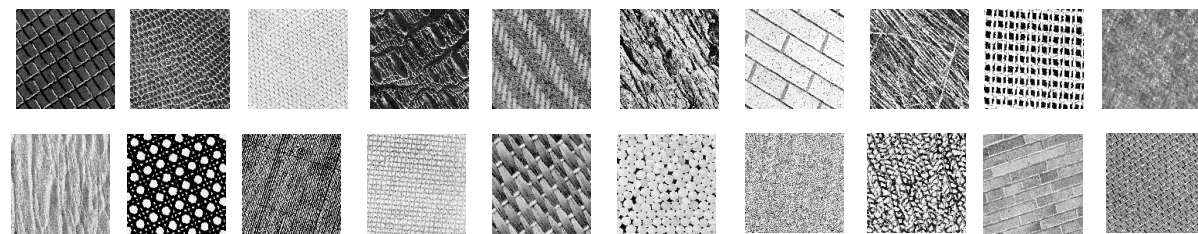

Figure 320 textures from Brodatz database used for affine invariant texture classification

$$
\begin{aligned}
& c_{a}\left(\theta_{a}\right)=\int\left(w_{1}-\overline{w_{1}}\right) F_{a}\left(w_{1}, \theta_{a}\right) d w_{1} \\
& c(\theta)=\int\left(w_{2}-\overline{w_{2}}\right) F\left(w_{2}, \theta\right) d w_{2}
\end{aligned}
$$

where $\overline{w_{1}}$ and $\overline{w_{2}}$ are the mean value of $w_{1}$ and $w_{2}$. Since the shift factor has been removed in Equation (9), it is obviously that $c_{a}\left(\theta_{a}\right)$ equals to $c(\theta)$. Notice that power spectrum also plays a very important role in measuring texture properties. We incorporate power spectrum that is invariant to scaling and compute the spectrum signatures at angle $\theta$ and $\theta_{a}$ as follows:

$$
\begin{aligned}
T\left(\theta_{a}\right) & =c_{a}\left(\theta_{a}\right) \int F_{a}\left(w_{1}, \theta_{a}\right) d w_{1} \\
& =c(\theta) \int F\left(w_{2}, \theta\right) d w_{2}=T(\theta)
\end{aligned}
$$

Such that we have obtained the orientation spectrum signatures $T(\theta)$ which is invariant to anisotropic scale (notice that affine transform results in different scaling along different orientations). This is the key problem of affine invariant texture analysis.

When we investigate the spectrum signatures along all the orientations throughout the whole image, $T(\theta)$ becomes the distribution function of spectrum signatures at different angles as illustrated in Figure 2. The relative larger peaks of $T(\theta)$ indicate those specific, dominating directions within the spectrum domain, which in turn exhibit stronger regular texture properties. While the signature distribution function changes under affine transform, the order of relevant larger peaks, their number, and height tend to be quite stable [3]. The PDV is constructed by those peaks. A peak $p(i)$ of $T(\theta)$ is defined as follows:

$$
p(i)=T\left(\theta_{i}\right)
$$

when $T\left(\theta_{i}\right)$ satisfy the following conditions

1. $T\left(\theta_{i}\right)=\max _{m \in w s}\left(T\left(\theta_{m}\right)\right)$ and $\theta_{i}$ is the center point of its local window size of $w s$ 


$$
\text { 2. } \int_{m \in w s}\left(T\left(\theta_{m}\right)-\frac{1}{w s} \int_{m \in w s} T\left(\theta_{m}\right) d \theta\right)^{2} d \theta \geq t
$$

where $t$ is a given threshold to select the peaks. Since the spacing between peaks can be small, the size of estimation window cannot be very large. Here we use the local window size of $1 \times 7$. A global view of the process of the PDV extraction is illustrated in Figure 2.

The feature vector is constructed by computing statistics of PDV. The statistics we used here are the largest value, the average value, the standard deviation, and the peak density. The peak density is calculated as the ratio between their number and the length of $\theta$.

\section{EXPERIMENTAL RESULTS}

In order to test the performance of the proposed features for affine invariant texture analysis, we carry out the experiment on 20 structured texture images selected from Brodatz album illustrated in Figure 3 (if textures are isotropic or randomness, any texture descriptors will be invariant to affine transform [8]). Each texture image of size $512 \times 512$ is affine transformed into 15 versions, from which subimages of size $128 \times 128$ are extracted. Thus a database of 300 images are constructed for this experiment. A database of 120 (6 for each texture class) are used for training and the remaining (180 images, 9 for each texture) for testing. The PDV is extracted from each image and the feature vector is constructed by computing statistics of PDV as mentioned in Section 3. Euclidean distance is calculated to measure the similarity between textures.

The K-nearest neighborhood classifier is employed. An average correct recognition rate of $88.33 \%$ is obtained. This clearly shows the promising efficacy of the proposed features for affine invariant texture classification.

\section{DISCUSSION AND CONCLUSIONS}

It has been reported that Gabor features and Wold features can characterize both ordered and random textures [4][8]. To the best of our knowledge, these texture models have not been developed for affine invariant texture classification. Our spectrum signatures are comparable to these features in measuring texture periodical properties. When Gabor masks are tuned to these orientations which our larger spectrum signatures indicate, the Gabor channels often produce larger output. For Wold features, the harmonic peaks that can measure texture structural properties are often centered on those regions around the orientations indicated by the peaks in $T(\theta)$. The discussions suggest that our features can capture texture properties as many other texture models. Furthermore they are invariant to affine transform.
The effectiveness of the proposed features on characterizing texture properties is theoretically justified in [5]. In fact, $\bar{w}$ in equation (10) is very similar to the carrier wave proposed in [5] and the spectrum signature is much like the AMPM component (amplitude modulation and phase modulation) which has been proved to efficiently describe image structure by Daugman et al. in [5].

In conclusion, a new approach based on texture spectrum signatures has been proposed and demonstrated to be effective in affine invariant texture classification. On the other hand this new texture model is comparable to other powerful texture models in measuring texture regular properties as discussed above. This is a very good start in affine invariant texture analysis. This approach should be extensible to perspective invariant texture classification. We intend to explore this in our future work.

\section{REFERENCE}

[1] T.N. Tan, "Geometric Transform Invariant Texture Analysis," SPIE. Vol. 2488, pp475-485, 1995.

[2] Jezekiel Ben-Arie, Zhiqian Wang, "Pictorial Recognition of Objects Employing Affine Invariance in the Frequency Domain," IEEE Trans. on PAMI, Vol. 20, No.6, June 1998.

[3] D. Chetnerikov, "Pattern Regularity as a Visual Key," Image and Vision computing 18 pp. 975-985, 2000.

[4] Fang Liu and Rosalind W. Picard, "Periodicity, Directionality, and Randomness: Wold Features for Image Modeling and Retrieval," IEEE Trans. on Pattern Analysis and Machine intelligence. Vol. 18, No. 7, July 1996.

[5] J. G. Daugman and C. J. Downing, "Demodulation, Predictive Coding, and Spatial Vision," Opt. Soc. Am. A, Vol. 12,No. 4, pp. 641-660, April 1995.

[6] J. G. Zhang and T.N. Tan, "Brief Review of Invariant Texture Analysis Methods", accepted by Pattern Recogniton, 2001, in press.

[7] S. Chang et al. "Texture Discrimination by Projective Invariants," Pattern Recognition Letters 5 pp. 337-342, 1987.

[8] T.N.Tan, "Rotation Invariant Texture Features and Their Use in Automatic Script Identification," IEEE Trans. Pattern Analysis and Machine Intelligence Vol.20, No.7, pp. 751-756, July 1998.

[9] Coloma Ballester and Manuel González, "Affine Invariant Texture Segmentation and Shape from Texture by Variational Methods," Journal of Mathematical Imaging and Vision 9, 141-171, 1998. 